\title{
Extensive or Restricted Ultrasound Protocols to Measure Carotid Intima-Media Thickness: Analysis of Completeness Rates and Impact on Observed Rates of Change Over Time
}

Sanne A. E. Peters, MSc, Hester M. den Ruijter, PhD, Mike K. Palmer, PhD, Diederick E. Grobbee, MD, PhD, John R. Crouse, III, MD, Daniel H. O'Leary, MD, Gregory W. Evans, MA, Joel S. Raichlen, MD, and Michiel L. Bots, MD, PhD, on Behalf of the METEOR Study Investigators, Utrecht, The Netherlands; Keele, United Kingdom; Winston-Salem, North Carolina; Boston, Massachusetts; Wilmington, Delaware

\begin{abstract}
Background: Ultrasound protocols to measure carotid intima-media thickness (CIMT) vary considerably with regard to carotid sites and angles that are assessed. Measurements from the carotid bifurcation and internal carotid artery are thought to be affected by large numbers of missing data. Actual published quantification of completeness rates and the relation with cardiovascular risk factors, however, is scarce. Also, it is currently unknown whether extensive ultrasound protocols including assessment of the carotid bifurcation and internal carotid artery add information in detecting rate of change in CIMT induced by drug therapy. These issues were addressed in this study using data from Measuring Effects on Intima-Media Thickness: An Evaluation of Rosuvastatin (METEOR).

Methods: In METEOR, carotid ultrasound examinations were performed twice before randomization, once each at 6,12 , and 18 months after randomization, and twice after 24 months of study treatment. B-mode ultrasound images were obtained from the near and far walls of the left and right common carotid artery, bifurcation, and internal carotid artery at five predefined angles. Completeness of CIMT data was assessed by carotid site and by angle. A site was considered complete when any of the five angles was measured. The relation between completeness at baseline and cardiovascular risk factors was assessed using logistic regression analyses. Ultrasound protocols with a reduced number of carotid sites and angles were retrospectively constructed, and differences in the rate of change in maximum CIMT between ultrasound protocols were compared.
\end{abstract}

Results: At each visit, CIMT measurements from all 12 carotid sites were available for $>94 \%$ of the participants. Incompleteness was the highest for near wall of the internal carotid artery and for the extreme angles $\left(60^{\circ}\right.$ and $\left.300^{\circ}\right)$. Of 12 risk factors examined, higher body mass index was related to incompleteness. Ultrasound protocols with a reduced number of angles resulted in similar estimates for the differences in rate of change in maximum CIMT. However, reductions in the number of sites gave results in the same direction but with different magnitudes and larger standard errors.

Conclusions: High levels of complete data can be obtained with extensive ultrasound protocols that include measurement from the carotid bifurcation and internal carotid artery. A high body mass index contributes to incompleteness of CIMT measurements. Extensive ultrasound protocols are required to obtain the highest precision to observe a treatment effect and to fully cover the degree of atherosclerotic burden. (J Am Soc Echocardiogr 2012;25:91-100.)

Keywords: Carotid intima-media thickness, Ultrasound protocols, Completeness rates, Trial design

From the Julius Center for Health Sciences and Primary Care, University Medical Center Utrecht, Utrecht, The Netherlands (S.A.E.P., H.M.R., D.E.G., M.L.B.); Keele University, Keele, United Kingdom (M.K.P.); the Department of Medicine, Wake Forest University School of Medicine, Winston-Salem, North Carolina (J.R.C., G.W.E.); Caritas Carney Hospital, Boston, Massachusetts (D.H.O.); and AstraZeneca, Wilmington, Delaware (J.S.R.)

Measuring Effects on Intima-Media Thickness: An Evaluation of Rosuvastatin was funded by AstraZeneca. Dr. Peters is supported by an unrestricted grant from AstraZeneca. Dr. Palmer was a former employee of AstraZeneca. Dr. Grobbee has received grant support from and delivered lectures for Pfizer, AstraZeneca, Organon, Servier, and Merck. Dr. Crouse has received grant or salary support from Merck, Merck-Schering Plough, Pfizer, AstraZeneca, and Kos Pharmaceuticals. He has delivered lectures for Merck, Merck-Schering Plough, Pfizer, AstraZeneca, Abbott, and Kos Pharmaceuticals. Dr. O'Leary serves on data safety and monitoring boards for Pfizer and AstraZeneca. He serves as a consultant to Pfizer,
Sankyo Pharma, Sanofi-Aventis, GlaxoSmithKline, Eli Lilly, Schering-Plough, Esperion Therapeutics, and Merck. He is an equity partner in Imagepace, LLC. Mr. Evans has received grant support and honoraria from AstraZeneca, Organon, and Pfizer and has served as a consultant for AstraZeneca and Pfizer. Dr. Raichlen is an employee of AstraZeneca. Dr. Bots has received study grants for studies on carotid intima-media thickness and/or honoraria for professional input on carotid intima-media thickness issues from AstraZeneca, Boeringer, Organon, Pfizer, Servier, and Unilever.

Reprint requests: Sanne A. E. Peters, Julius Center for Health Sciences and Primary Care, Stratenum 6.131, University Medical Center Utrecht, Heidelberglaan 100, 3584 CX, Utrecht, The Netherlands (E-mail: s.a.e.peters@umcutrecht.n/).

0894-7317/ $\$ 36.00$

Copyright 2012 by the American Society of Echocardiography.

doi:10.1016/j.echo.2011.09.009 


\begin{tabular}{|l|}
\hline \multicolumn{1}{|c|}{ Abbreviations } \\
\hline BMI = Body mass index \\
CIMT = Carotid intima-media \\
thickness \\
METEOR = Measuring \\
Effects on Intima-Media \\
Thickness: An Evaluation of \\
Rosuvastatin \\
RADIANCE = Rating \\
Atherosclerotic Disease \\
Change by Imaging With \\
a New CETP Inhibitor \\
\hline
\end{tabular}

Carotid intima-media thickness (CIMT) is commonly used in observational studies to examine determinants and consequences of atherosclerosis. ${ }^{1-5}$ In addition, changes in CIMT over time are increasingly being used in clinical trials as an alternative end point for cardiovascular morbidity and mortality to study the efficacy of pharmacologic interventions. ${ }^{6}$ The main advantage of the use of CIMT as a primary outcome rather than hard end points, such as cardiovascular morbidity and mortality, is the reduction in costs due to a smaller sample size and shorter duration of follow-up. This may result in earlier detection of the presence or absence of effects of drug therapies on atherosclerotic progression. ${ }^{7}$

At present, little uniformity exists on ultrasound protocols across studies. Some studies have used extensive protocols, including measurements from the near and far walls of the common carotid artery, carotid bifurcation, and internal carotid artery at multiple angles of interrogation, ${ }^{8-11}$ whereas others have restricted their protocols by measuring only the far wall of the common carotid artery. ${ }^{12-14}$ The thickest sites of the carotid artery are typically found in the internal carotid artery and in the carotid bifurcation. Difficulties in visualizing these sites in general, and near wall sites in particular, are thought to give rise to large amounts of missing data. One of the main arguments to restrict ultrasound protocols to measurements of the far wall of common carotid artery is that common CIMT data collection is nearly always complete in this segment. ${ }^{7}$ However, data on the completeness of CIMT measurements are often not reported, and if reported, only overall completeness rates are provided. ${ }^{10,15-17}$ Also, cross-sectional observed differences in absolute thickness across carotid segments do not necessarily result in a difference in observed rate of change over time. A thorough evaluation of whether observed rates of change in CIMT differ across ultrasound protocols that vary in the numbers of segments, sites, and angles assessed is lacking.

In contrast to the general view in the field, a recent analysis demonstrated that high levels of complete CIMT data could be obtained with extensive ultrasound protocols that also included measurements of the near wall of the carotid bifurcation and internal carotid artery. ${ }^{18}$ The investigators found that body mass index (BMI) and waist circumference were the main predictors of incomplete data. ${ }^{18}$

Because there is still no agreement on the optimal ultrasound protocol in terms of high completeness rates and the evidence on this issue is limited, we studied the completeness of CIMT data and its relation with cardiovascular risk factors using data from a randomized controlled trial that used an extensive ultrasound protocol. Because completeness alone should not be the main argument to use an extensive or a restricted ultrasound protocol, we subsequently studied the differences between extensive and restricted ultrasound protocols in relation to rate of change in CIMT.

\section{METHODS}

\section{Study Population}

In this post hoc analysis, data from the Measuring Effects on IntimaMedia Thickness: An Evaluation of Rosuvastatin (METEOR) trial.
The rationale, design, and main outcomes of this study have been de scribed in detail elsewhere. ${ }^{10,19}$ Briefly, METEOR was a randomized, double-blind, placebo-controlled trial among 984 individuals at low risk for cardiovascular disease on the basis of 10-year Framingham risk of $<10 \%$ but with modest increases in CIMT as evidenced by at least one maximum CIMT measurement $\geq 1.2 \mathrm{~mm}$ and no measurement $\geq 3.5 \mathrm{~mm}$ from two separate ultrasound examinations in any of the 12 carotid segments assessed (described below). The main objective of METEOR was to assess the impact of rosuvastatin $40 \mathrm{mg} /$ day versus placebo on the rate of change in CIMTover 2 years of follow-up.

\section{B-Mode Ultrasound Method}

Ultrasound examinations were performed twice before randomization; once each at 6, 12, and 18 months after randomization; and then twice at the end of 24 months of study treatment. At each visit, ultrasound images were obtained from six well-defined arterial segments from both the left and right carotid artery (Figure 1), as detailed elsewhere. ${ }^{19}$ These segments were defined as the near and far walls of the common carotid artery extending from 10 to $20 \mathrm{~mm}$ proximal to the tip of the flow divider; the near and far walls of the carotid bifurcation as assessed from the tip of the flow divider, extending $10 \mathrm{~mm}$ proximal to the tip of the flow divider; and the near and far walls of the internal carotid artery as assessed in the $10 \mathrm{~mm}$ distal to the tip of the flow divider. There was no minimal acceptable length for a measurement, and a measurement was accepted as long as the reader had enough confidence to mark the CIMT. In METEOR, all images were measured in end-diastole at the top of the $\mathrm{R}$ wave of the electrocardiogram. The head of each participant was rotated $45^{\circ}$ to the other side to better expose each carotid artery for interrogation. The Meijer carotid arc was used to image the artery at five prespecified angles in steps of $30^{\circ}$ (from $60^{\circ}$ to $180^{\circ}$ on the right carotid artery and from $300^{\circ}$ to $180^{\circ}$ on the left carotid artery). This resulted in a maximum of 60 sets of CIMT measurements that could be provided at each examination.

The equipment used in METEOR for CIMT imaging was the Acuson Sequoia 512, Acuson Sequoia 256, and SONOLINE Antares (all Siemens Medical Solutions USA, Inc., Mountain View, CA) and the HDI 5000 (Phillips Medical Systems, Andover, MA). These devices all have electrocardiographic gating, and all used high-resolution linear-array probes around 7.5 MHz.

These systems were all approved by a quality control center, and the performance of these systems was monitored using phantoms. The real-time captured images were all stored on S-VHS videotape and were sent to one of two specialized ultrasound reading centers in Europe (University Medical Centre, Utrecht, The Netherlands) and the United States (Wake Forest University School of Medicine, Winston-Salem, NC) for CIMT measurement. All images were digitized and read at these two at reading centers.

All ultrasound scans were manually read with Image Pro software (Microsoft Corporation, Redmond, WA). Images were read by 16 readers who completed a uniform training program that ensured standardized settings across reading stations and core laboratories. Readers traced trailing edges on the near wall boundaries and leading edges on the far wall boundaries and were free to set as many anchors as needed to trace the interface, with straight lines connecting the anchor points. All images were read in a blinded batch fashion after each participant had finished. A batch consisted of all images collected from a single participant and was read by a single reader over an interval of a few days with visits arranged in random order to minimize 


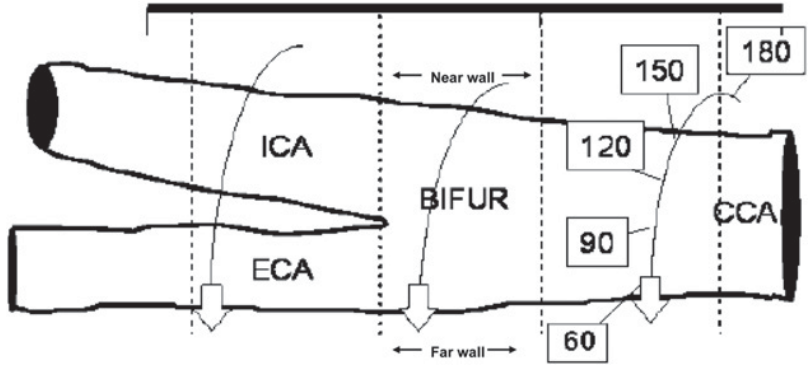

Figure 1 Graphical representation of circumferential assessment of the artery sites. BIFUR, Carotid bifurcation; CCA, common carotid artery; $E C A$, external carotid artery; ICA, internal carotid artery.

the impact of between-reader variability and temporal drift on estimates of change in CIMT within participants.

\section{Laboratory Analyses}

As previously described, blood samples were taken for analysis of serum lipids, lipoproteins, and C-reactive protein. ${ }^{10}$ The samples were sent immediately for analysis to one of two central standardized laboratories in Europe (Covance Central Laboratory Services, Geneva, Switzerland) and in the United States (Covance Central Laboratory Services, Indianapolis, IN). Baseline samples were used for the present analysis.

\section{Variations in Ultrasound Protocols}

We simulated eight different ultrasound protocols that may be considered once designing a CIMT trial by selectively excluding up to $83 \%$ of the specific carotid segments and carotid walls. The most extensive ultrasound protocol was the original METEOR protocol, which included assessment of the near and far walls of the left and right common carotid artery, carotid bifurcation, and internal carotid artery. In the most restricted ultrasound protocol, only measurements from the far wall of the left and right carotid artery were performed. Each of the eight ultrasound protocols was applied to settings in which five, three, or one circumferential carotid angle was measured. Angle-specific measurements in ultrasound protocols with assessment of five carotid angles were performed at $60^{\circ}, 90^{\circ}, 120^{\circ}, 150^{\circ}$, and $180^{\circ}$ on the right carotid artery and for the left carotid artery at $300^{\circ}, 270^{\circ}, 240^{\circ}, 210^{\circ}$, and $180^{\circ}$. Ultrasound protocols with three circumferential CIMT measurements for each carotid site were performed at $90^{\circ}, 120^{\circ}, 150^{\circ}$ for the right carotid artery and at $270^{\circ}, 240^{\circ}$, and $210^{\circ}$ for the left carotid artery. CIMT measurements were performed at $120^{\circ}$ and $240^{\circ}$ of the right and left carotid artery, respectively, in protocols in which only one carotid angle was evaluated for each carotid segment. The primary end point for this analysis was the annualized rate of change in the mean of the maximum CIMT measurements based on all scans performed during the 2-year study period from each of the 12 carotid artery sites. The maximum CIMT in each of the 12 sites is defined as the largest measurement derived from the assessed interrogation angles, each one $30^{\circ}$ different from the adjacent angle (i.e., the number of CIMT values used for the model fit was maximally 12 for each visit, irrespective of the number of angles assessed). When a carotid plaque was present, it was included in this measurement.

\section{Statistical Analyses}

Completeness rates were evaluated at the level of the carotid angle for the left and right carotid artery separately (five angles each), and

\begin{tabular}{|c|c|c|}
\hline Variable & $\begin{array}{l}\text { Rosuvastatin } \\
(n=702)\end{array}$ & $\begin{array}{l}\text { Placebo } \\
(n=282)\end{array}$ \\
\hline Age (y) & $57 \pm 6.2$ & $57 \pm 6.0$ \\
\hline Men & $421(60 \%)$ & 167 (59\%) \\
\hline Caucasian & 659 (94\%) & $268(95 \%)$ \\
\hline $\mathrm{BMI}\left(\mathrm{kg} / \mathrm{m}^{2}\right)$ & $27.1 \pm 4.0$ & $27.5 \pm 4.0$ \\
\hline $\begin{array}{l}\text { Smoking (during the previous } \\
\text { month) }\end{array}$ & $22(3 \%)$ & $16(6 \%)$ \\
\hline Family history of premature $\mathrm{CHD}^{*}$ & $65(9 \%)$ & $31(11 \%)$ \\
\hline Hypertension $^{\dagger}$ & $138(20 \%)$ & $58(21 \%)$ \\
\hline Total cholesterol (mg/dL) & $229 \pm 28.7$ & $230 \pm 27.7$ \\
\hline $\begin{array}{l}\text { LDL cholesterol }(\mathrm{mg} / \mathrm{dL})^{\ddagger} \\
\mathrm{HDL} \text { cholesterol }(\mathrm{mg} / \mathrm{dL})^{\ddagger}\end{array}$ & $\begin{array}{c}155 \pm 24.1 \\
50 \pm 9.0\end{array}$ & $\begin{array}{c}154 \pm 24.2 \\
49 \pm 9.2\end{array}$ \\
\hline Triglycerides (mg/dL) & $126 \pm 64.3$ & $134 \pm 67.8$ \\
\hline $\mathrm{CRP}(\mathrm{mg} / \mathrm{L})$ & $1.40(0.80-2.90)$ & $1.60(0.80-3.20)$ \\
\hline $\begin{array}{l}\text { Mean of the maximum of all } 12 \\
\text { CIMT sites }(\mathrm{mm})\end{array}$ & $1.15 \pm 0.19$ & $1.17 \pm 0.20$ \\
\hline Mean CIMT of the $\mathrm{CCA}^{\S}(\mathrm{mm})$ & $0.76 \pm 0.12$ & $0.76 \pm 0.12$ \\
\hline
\end{tabular}

$C C A$, Common carotid artery; $C H D$, coronary heart disease; $C R P$ C-reactive protein; $H D L$, high-density lipoprotein; $L D L$, low-density lipoprotein.

Data are expressed as mean $\pm \mathrm{SD}$, as number (percentage), or as median (interquartile range).

${ }^{*}$ Defined as CHD in a first-degree male relative aged $<55$ years or in a first-degree female relative aged $<65$ years.

${ }^{\dagger}$ Blood pressure $\geq 140 / 90 \mathrm{~mm} \mathrm{Hg}$ or taking antihypertensive medication.

${ }^{\ddagger}$ To convert HDL and LDL cholesterol to millimoles per liter, multiply by 0.0259 .

${ }^{\S}$ Average of the mean CIMT of the four CCA sites: near and far walls of the right and left CCA.

carotid site (12 sites). Completeness of the angle-specific measurements is expressed as the average completeness on the six carotid sites that were examined on both the left and right carotid artery. Sitespecific measurements were considered available when at least one CIMT measurement at any of the five angles was performed. This definition was used because in METEOR, the availability of at least one of five angle-specific measurements was required to have a site-specific CIMT value. All availability rates are presented as percentages. The numerator for completeness was the number of available CIMT measurements, and the denominator for completeness at a specific visit consisted of all those participants who indeed underwent carotid ultrasound examinations at that visit. Subgroup analyses were performed to examine differences in completeness for participants with BMIs $\geq 30 \mathrm{~kg} / \mathrm{m}^{2}$ and to study differences between European participants and participants from the United States.

Logistic regression analyses were used to study the relation between completeness of CIMT measurements at the site level at two baseline measurements and cardiovascular risk factors. Risk factors included were age, sex, BMI, alcohol use, family history of coronary heart disease (in a first-degree male relative aged $<55$ years or in a first-degree female relative aged $<65$ years), hypertension (blood pressure $\geq 140 / 90 \mathrm{~mm} \mathrm{Hg}$ or taking antihypertensive medication), smoking during the previous month, low-density lipoprotein cholesterol, high-density lipoprotein cholesterol, triglycerides, C-reactive protein, and maximum CIMT. The levels of the risk factors were restricted to baseline measurements. Analyses were repeated with 

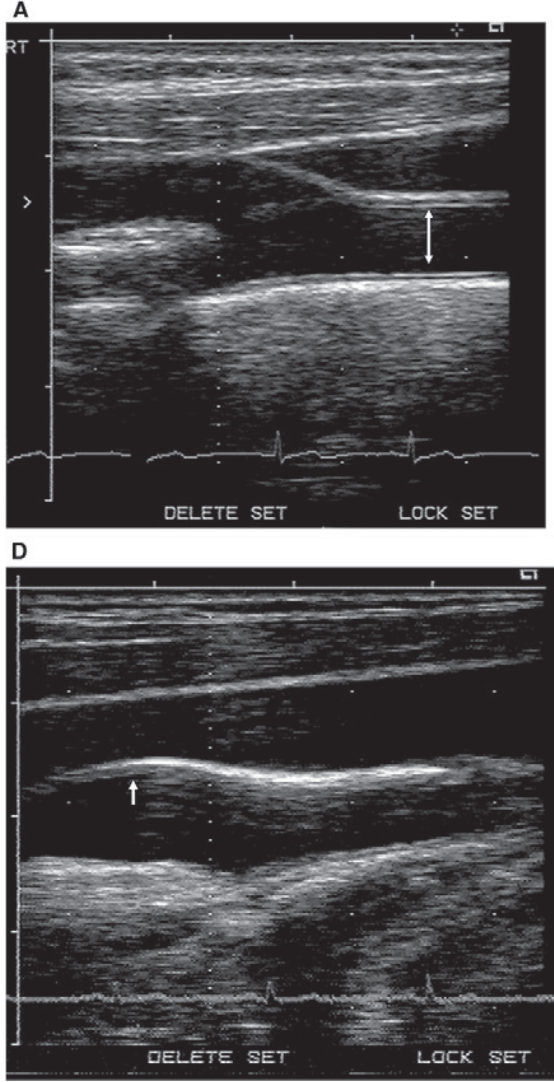

B
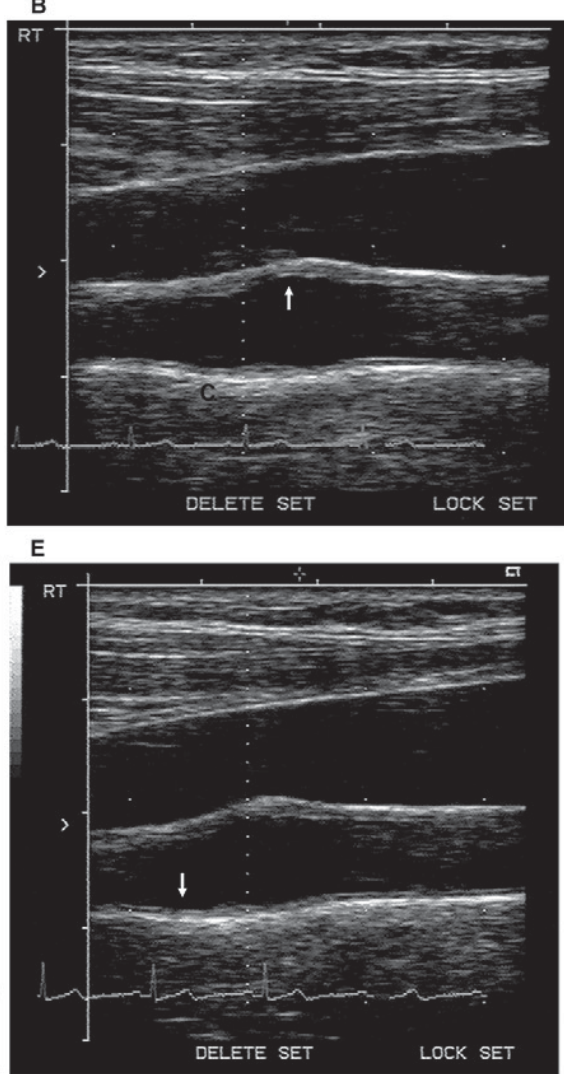

C

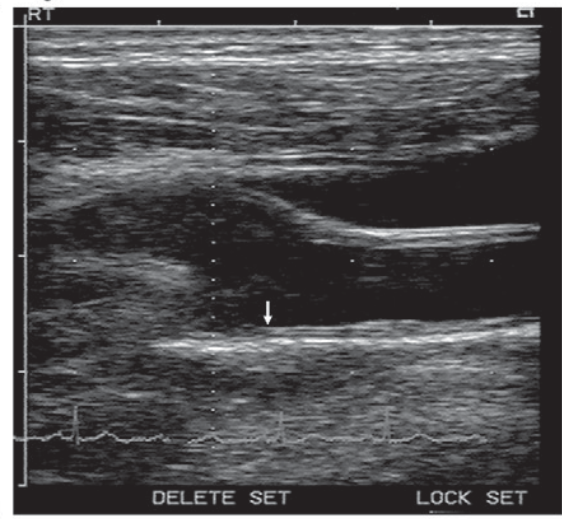

Figure 2 Longitudinal B-mode images of the carotid artery showing clear interfaces for measurement of CIMT multiple carotid segments. Arrows indicate the locations where CIMT measurements were performed. (A) Near and far walls of the common carotid artery. (B) Near wall of the carotid bifurcation. (C) Far wall of the carotid bifurcation. (D) Near wall of the internal carotid artery. (E) Far wall of the internal carotid artery.

adjustments for age, sex, and BMI, because these variables were considered to confound the univariate associations. Results of the adjusted and unadjusted analyses are presented as odds ratios (ORs) and corresponding 95\% confidence intervals (CIs).

The rate of change in CIMT in each of the ultrasound protocols considered was evaluated by a multilevel, repeated-measures, linear mixed-effects model using the lme 4 package available in $\mathrm{R}$ statistical software. ${ }^{20}$ This analysis was similar to the analysis performed in the original METEOR study. ${ }^{10}$ The levels used were defined by (1) the participant and (2) the carotid artery site within the participant. The repeated measure was time. The model was specified in terms of fixed effects for carotid artery site, age, sex, scan reader, ultrasound machine, treatment group, time, and the interaction between treatment group and time. Time as a continuous variable was the interval from the date of randomization to the date of CIMT measurement. Random effects within the model were intercept and slope for individual participants and for sites within participants.

\section{RESULTS}

The baseline characteristics of the study participants by treatment allocation are presented in Table 1 . The mean age of the participants was $57 \pm 6$ years, the majority were men $(60 \%)$, and the mean BMI was $27 \pm 4.0 \mathrm{~kg} / \mathrm{m}^{2}$. Figures $2 \mathrm{~A}$ to $2 \mathrm{E}$ provide example longitudinal B-mode images of the carotid artery showing clear interfaces for measurements of CIMT in the near and far walls of the common carotid artery, carotid bifurcation, and internal carotid artery.

\section{Completeness at the Site Level}

Table 2 shows the rates of completeness that were obtained on each of the 12 carotid sites. The percentage of CIMT measurements at the baseline examinations was $94 \%$ for the near wall of the right internal carotid artery and $96 \%$ for the near wall of the left internal carotid artery. Completeness on the other carotid artery sites, including the carotid bifurcation, was $>99 \%$.

The number of participants who withdrew from the study, who did not undergo ultrasound examinations and hence had no CIMT data, was nine $(1 \%)$ for the 6 -month visit, $52(6 \%)$ for the 12 -month visit, $110(13 \%)$ for the 18-month visit, $133(15 \%)$ for the first 24-month visit, and 160 (18\%) for the second 24-month visit. Of those with ultrasound examinations after 2 years of follow-up, 95\% of the near wall of the right internal carotid artery measurements and $97 \%$ of the near wall of the left internal carotid artery measurements were complete. Complete CIMT data on the other carotid sites was available in $99 \%$ of the subjects who underwent end-of-study ultrasound examinations.

Completeness rates for the near wall of the left and right internal carotid artery were lower than those of the far wall of the internal carotid artery. No major differences $(\leq 0.5 \%)$ were present between the near and far walls of the right or left common carotid artery or carotid bifurcation during all visits.

Analyzing participants from Europe and the United States separately showed a similar completeness rate of $>99 \%$ for all carotid sites, except for the near wall of the internal carotid artery. Completeness for the near wall of the right internal carotid artery 
Table 2 Percentages of available CIMT measurements by visit (rows) and carotid artery sites (columns)

\begin{tabular}{|c|c|c|c|c|c|c|c|c|c|c|c|c|}
\hline Weeks after randomization & LCF & LCN & LBF & LBN & LIF & LIN & RCF & RCN & RBF & RBN & RIF & RIN \\
\hline-4 & $>99.9$ & 99.9 & $>99.9$ & 99.7 & 99.7 & 96.2 & $>99.9$ & $>99.9$ & 99.8 & 99.9 & 99.1 & 93.9 \\
\hline-2 & $>99.9$ & 99.9 & 99.8 & 99.4 & 99.4 & 96.2 & 99.9 & 99.9 & 99.9 & $>99.9$ & 99.4 & 94.2 \\
\hline 26 & $>99.9$ & 99.9 & 99.8 & 99.7 & 99.5 & 96.7 & 99.9 & 99.9 & $>99.9$ & 99.9 & 99.2 & 95.0 \\
\hline 52 & 99.9 & 99.6 & 99.8 & 99.4 & 99.2 & 97.5 & $>99.9$ & $>99.9$ & $>99.9$ & 99.9 & 99.5 & 94.8 \\
\hline 78 & 99.9 & 99.9 & 99.9 & 99.2 & 98.8 & 96.0 & $>99.9$ & 99.7 & $>99.9$ & 99.9 & 99.2 & 95.0 \\
\hline 104 & $>99.9$ & 99.7 & 99.6 & 99.6 & 99.5 & 96.8 & $>99.9$ & 99.9 & $>99.9$ & 99.7 & 99.7 & 95.2 \\
\hline 105 & $>99.9$ & 99.7 & 99.9 & 99.4 & 98.9 & 97.2 & $>99.9$ & $>99.9$ & 99.9 & 99.9 & 99.3 & 95.8 \\
\hline
\end{tabular}

$\angle B F$, Far wall of the left bifurcation; $L B N$, near wall of the left bifurcation; $L C F$, far wall of the left common carotid artery; $L C N$, near wall of the left common carotid artery; LIF, far wall of the left internal carotid artery; LIN, near wall of the left internal carotid artery; RBF, far wall of the right bifurcation; $R B N$, near wall of the right bifurcation; $R C F$, far wall of the right common carotid artery; $R C N$, near wall of the right common carotid artery; $R I F$, far wall of the right internal carotid artery; $R I N$, near wall of the right internal carotid artery.

Table 3 Percentages of available CIMT measurements over six carotid sites at the angle level for each ultrasound examination

\begin{tabular}{cccccccc}
\hline & \multicolumn{7}{c}{ Weeks after randomization } \\
\cline { 2 - 8 } Angle & -4 & -2 & 26 & 52 & 78 & 104 & 105 \\
\hline \multicolumn{7}{c}{ Left carotid artery } \\
$180^{\circ}$ & 82.7 & 81.9 & 83.0 & 83.7 & 82.6 & 82.9 & 83.7 \\
$210^{\circ}$ & 88.3 & 88.7 & 88.8 & 89.9 & 89.4 & 89.1 & 89.8 \\
$240^{\circ}$ & 93.2 & 93.7 & 92.9 & 93.6 & 92.3 & 93.6 & 94.2 \\
$270^{\circ}$ & 87.6 & 88.3 & 87.6 & 88.3 & 87.6 & 86.4 & 86.5 \\
$300^{\circ}$ & 37.5 & 39.0 & 38.5 & 37.3 & 33.9 & 31.2 & 33.1 \\
\hline Right carotid artery & & & & & & \\
$180^{\circ}$ & 82.8 & 82.9 & 84.4 & 84.4 & 82.1 & 83.2 & 84.2 \\
$150^{\circ}$ & 89.1 & 88.7 & 89.7 & 89.3 & 89.7 & 90.5 & 89.4 \\
$120^{\circ}$ & 92.7 & 93.8 & 93.5 & 92.7 & 93.2 & 93.1 & 93.1 \\
$90^{\circ}$ & 90.0 & 90.3 & 91.3 & 82.7 & 89.8 & 89.2 & 90.7 \\
$60^{\circ}$ & 49.5 & 49.8 & 50.7 & 54.0 & 42.4 & 39.5 & 40.7 \\
\hline
\end{tabular}

Availability results represent average availability for the near and far walls of the common carotid artery, carotid bifurcation, and internal carotid artery.

over time was on average $97 \%$ for European participants and $93 \%$ for participants from the United States. For the left internal carotid artery, $97 \%$ of the measurements were available for European participants, and $96 \%$ of the measurements were available for participants from the United States.

Availability of CIMT data in participants with BMIs $\geq 30 \mathrm{~kg} / \mathrm{m}^{2}$ was $90 \%$ for the near wall of the right internal carotid artery and $95 \%$ for the near wall of the left internal carotid artery. Completeness rates for all other carotid sites were similar to the analyses on all subjects.

\section{Completeness at the Angle Level}

The availability of CIMT measurements was the highest at $240^{\circ}$ of the left carotid artery and at $120^{\circ}$ of the right carotid artery (Table 3 ). At these angles, 93\% of the CIMT measurements were available at baseline for both carotid arteries, with 94\% available CIMT measurement at the end of follow-up for the left carotid artery and 93\% for the right carotid artery. The lowest number of available measurements was found at the most extreme angles, which were $300^{\circ}$ for the left ca-
Table 4 ORs ( $95 \%$ Cls) of the determinants of completeness of CIMT measurements

\begin{tabular}{|c|c|c|c|}
\hline Variable & Unadjusted & $\begin{array}{l}\text { Age and sex } \\
\text { adjusted }^{\ddagger}\end{array}$ & $\begin{array}{l}\text { Age, sex, and } \\
\text { BMI adjusted }\end{array}$ \\
\hline Age & $0.98(0.95-1.01)$ & $1.00(0.97-1.04)$ & $0.98(0.95-1.01)$ \\
\hline Male gender & $0.65(0.45-0.94)$ & $0.65(0.42-1.00)$ & $0.69(0.47-1.01)$ \\
\hline BMI & $0.87(0.84-0.91)$ & $0.88(0.84-0.92)$ & NA \\
\hline Alcohol use & $0.64(0.44-0.93)$ & $0.68(0.46-0.99)$ & $0.68(0.46-1.01)$ \\
\hline $\begin{array}{c}\text { Family history } \\
\text { of } \mathrm{CHD}^{*}\end{array}$ & $1.42(0.80-2.55)$ & $1.30(0.72-2.36)$ & $1.29(0.69-2.40)$ \\
\hline Hypertension $^{\dagger}$ & $1.53(0.99-2.36)$ & $1.30(0.72-2.36)$ & $1.13(0.71-1.81)$ \\
\hline Smoking & $1.66(0.70-3.94)$ & $1.37(0.57-3.35)$ & $1.89(0.76-4.67)$ \\
\hline LDL cholesterol & $1.05(0.78-1.41)$ & $1.05(0.78-1.41)$ & $1.03(0.76-1.41)$ \\
\hline HDL cholesterol & $1.28(0.58-2.83)$ & $1.71(0.74-3.95)$ & $1.05(0.44-2.51)$ \\
\hline Triglycerides & $0.75(0.60-0.94)$ & $0.73(0.58-0.91)$ & $0.86(0.67-1.10)$ \\
\hline CRP & $0.73(0.55-0.98)$ & $0.78(0.58-1.06)$ & $1.01(0.70-1.45)$ \\
\hline Maximum CIMT & $0.86(0.61-1.21)$ & $0.87(0.61-1.22)$ & $0.86(0.61-1.23)$ \\
\hline
\end{tabular}

$C H D$, Coronary heart disease; $C R P$, C-reactive protein; $H D L$, highdensity lipoprotein; $L D L$, low-density lipoprotein.

*Defined as CHD in a first-degree male relative aged $<55$ years or in a first-degree female relative aged $<65$ years.

${ }^{\dagger}$ Blood pressure $\geq 140 / 90 \mathrm{~mm} \mathrm{Hg}$ or taking antihypertensive medication.

${ }^{\ddagger}$ Age was adjusted for sex, and sex was adjusted for age.

${ }^{\S}$ Age was adjusted for sex and BMI, and sex was adjusted for age and BMI.

rotid artery and $60^{\circ}$ for the right carotid artery. At the beginning of the study, $38 \%$ of the CIMT measurements at $300^{\circ}$ were available for the left carotid artery, and $50 \%$ of the measurements at $60^{\circ}$ were available for the right carotid artery. Furthermore, completeness rates were generally lower in the left carotid artery than in the right carotid artery. The average completeness rates over all ultrasound examinations for the $180^{\circ}, 210^{\circ}$, and $270^{\circ}$ angles of the left carotid artery were $83 \%, 89 \%$, and $87 \%$, respectively. For the right carotid artery, the completeness rates for the $180^{\circ}, 150^{\circ}$, and $90^{\circ}$ angles were $83 \%, 89 \%$, and $89 \%$, respectively.

\section{Determinants of Completeness}

The completeness rate at baseline for carotid sites was highly right skewed. The majority of participants (84\%) did have CIMT data for all sites at baseline. Three participants $(0.3 \%)$ had no data at five sites, 
Table 5 Annualized rate of change in mean maximum CIMT by treatment allocation with measurements from five, three, or one angle at a maximum of 12 carotid artery sites

\begin{tabular}{|c|c|c|c|}
\hline \multirow[b]{2}{*}{ Sites measured $(n)$} & \multicolumn{3}{|c|}{ Rate of change in CIMT (SE) $(\mathrm{mm} / \mathrm{y})$} \\
\hline & Rosuvastatin & Placebo & Rosuvastatin vs placebo \\
\hline \multicolumn{4}{|l|}{ Five angles } \\
\hline CCA, BIF, and ICA, NFW (60) & $-0.0014(0.0014)$ & $0.0131(0.0022)$ & $-0.0145(0.0026)$ \\
\hline CCA, NFW (20) & $-0.0038(0.0013)$ & $0.0084(0.0021)$ & $-0.0122(0.0025)$ \\
\hline CCA and BIF, NFW (40) & $-0.0039(0.0015)$ & $0.0126(0.0024)$ & $-0.0166(0.0028)$ \\
\hline CCA and ICA, NFW (40) & $0.0000(0.0014)$ & $0.0112(0.0023)$ & $-0.0112(0.0027)$ \\
\hline CCA, FW (10) & $-0.0056(0.0017)$ & $0.0045(0.0027)$ & $-0.0101(0.0032)$ \\
\hline CCA and BIF, FW (20) & $-0.0045(0.0021)$ & $0.0112(0.0033)$ & $-0.0157(0.0040)$ \\
\hline CCA and ICA, FW (20) & $0.0012(0.0019)$ & $0.0092(0.0030)$ & $-0.0081(0.0036)$ \\
\hline CCA, BIF, and ICA, FW (30) & $-0.0004(0.0019)$ & $0.0119(0.0030)$ & $-0.0123(0.0036)$ \\
\hline \multicolumn{4}{|l|}{ Three angles } \\
\hline CCA, BIF, and ICA, NFW (60) & $-0.0008(0.0014)$ & $0.0139(0.0022)$ & $-0.0147(0.0026)$ \\
\hline CCA, NFW (20) & $-0.0029(0.0013)$ & $0.0099(0.0020)$ & $-0.0128(0.0023)$ \\
\hline CCA and BIF, NFW (40) & $-0.0029(0.0015)$ & $0.0133(0.0024)$ & $-0.0162(0.0028)$ \\
\hline CCA and ICA, NFW (40) & $0.0002(0.0014)$ & $0.0126(0.0022)$ & $-0.0124(0.0026)$ \\
\hline CCA, FW (10) & $-0.0053(0.0017)$ & $0.0046(0.0026)$ & $-0.0099(0.0031)$ \\
\hline CCA and BIF, FW (20) & $-0.0040(0.0021)$ & $0.0103(0.0033)$ & $-0.0143(0.0039)$ \\
\hline CCA and ICA, FW (20) & $0.0006(0.0018)$ & $0.0092(0.0028)$ & $-0.0087(0.0033)$ \\
\hline CCA, BIF, and ICA, FW (30) & $-0.0005(0.0018)$ & $0.0114(0.0029)$ & $-0.0119(0.0034)$ \\
\hline \multicolumn{4}{|l|}{ One angle } \\
\hline CCA, BIF, and ICA, NFW (60) & $-0.0026(0.0013)$ & $0.0147(0.0021)$ & $-0.0173(0.0024)$ \\
\hline CCA, NFW (20) & $-0.0014(0.0014)$ & $0.0109(0.0022)$ & $-0.0122(0.0026)$ \\
\hline CCA and BIF, NFW (40) & $-0.0038(0.0015)$ & $0.0132(0.0024)$ & $-0.0170(0.0028)$ \\
\hline CCA and ICA, NFW (40) & $-0.0006(0.0014)$ & $0.0143(0.0022)$ & $-0.0149(0.0026)$ \\
\hline CCA, FW (10) & $-0.0045(0.0018)$ & $0.0068(0.0029)$ & $-0.0113(0.0034)$ \\
\hline CCA and BIF, FW (20) & $-0.0048(0.0020)$ & $0.0119(0.0032)$ & $-0.0167(0.0038)$ \\
\hline CCA and ICA, FW (20) & $-0.0009(0.0019)$ & $0.0116(0.0030)$ & $-0.0125(0.0035)$ \\
\hline CCA, BIF, and ICA, FW (30) & $-0.0022(0.0017)$ & $0.0135(0.0027)$ & $-0.0158(0.0032)$ \\
\hline
\end{tabular}

$B I F$, Carotid bifurcation; $C C A$, common carotid artery; FW, far wall; ICA, internal carotid artery; NFW, near and far walls.

three $(0.3 \%)$ had no data at four sites, eight $(0.9 \%)$ had no data at three sites, $38(4 \%)$ had no data at two sites, and $82(9 \%)$ had no data at one site.

Table 4 shows the determinants of arterial-site completeness at the prerandomization visits. Univariate analyses showed that male sex, higher BMI, alcohol use, higher levels of triglycerides, and higher Creactive protein levels were associated with a lower number of available CIMT measurements. When adjusted for age and sex, a higher BMI (OR, 0.88; 95\% CI, 0.84-0.92), alcohol use (OR, 0.68; 95\% CI, 0.46-0.99), and a higher level of triglycerides (OR, 0.73; $95 \%$ CI, 0.58-0.91) remained associated with a lower number of CIMT measurements. When adjusted for age, sex, and BMI, the association between the number of sites with CIMT measurements and alcohol use and triglyceride level, respectively, were substantially attenuated, with $95 \%$ Cls that included 1.

\section{Rate of Change in CIMT Across Ultrasound Protocols}

The annualized rates of change in CIMT for each of the eight ultrasound protocols considered in which five angles were measured are shown in Table 5. Findings on rate of change in maximum CIMT between treatment groups were largely in the same direction across the ultrasound protocols (i.e., nonsignificant regression in the rosuvastatin group and significant progression in the placebo group) and with statistically significant differences between treatment groups (Table 5).
However, protocols in which both the near and far walls were assessed consistently had the smallest standard errors, indicating the highest precision, compared with ultrasound protocols in which only the far walls were assessed. In addition, the magnitudes of the treatment effects different across the ultrasound protocols depending on which carotid segments were assessed. The detected rates of change were the largest in protocols that included the carotid bifurcation, whereas rates of change in CIMT were smallest in protocols that included only the common carotid artery.

Figure 3 shows the difference between rosuvastatin and placebo in rate of change in CIMT across the eight ultrasound protocols in which the number of angles assessed differed from five to three or one angle. The direction, magnitude, and precision of these rates of change were similar within ultrasound protocols that assessed the same carotid segments but measured five, three, or one angle.

\section{DISCUSSION}

The present study demonstrates that high levels of complete CIMT data were obtained using an extensive ultrasound protocol that includes measurements from the near wall of the carotid bifurcation and internal carotid artery. Rates of change in CIMT differed in magnitude, but not in direction, when limited ultrasound protocols were 

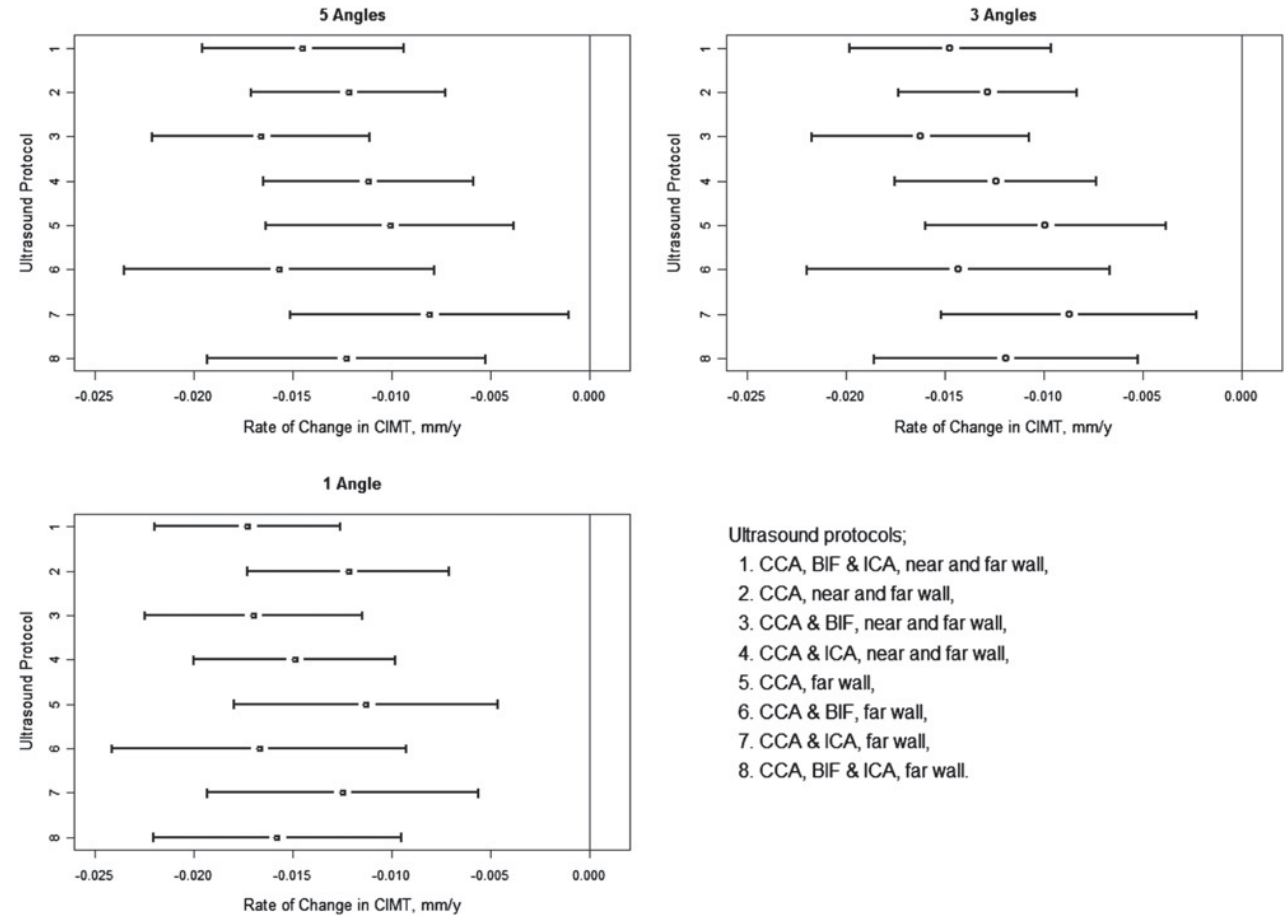

Figure 3 Difference between rosuvastatin and placebo in rate of change in maximum CIMT across ultrasound protocols and $95 \%$ Cls. BIF, Carotid bifurcation; CCA, common carotid artery; ICA, internal carotid artery.

used. Also, extensive ultrasound protocols were more precise to observe treatment effects than restricted protocol approaches. Hence, our completeness findings challenge the general view that measurements from the near wall of the carotid bifurcation and internal carotid artery are difficult to achieve and are a major source of missing data. Moreover, our data show that ultrasound protocols including near and far wall assessments at multiple carotid segments provide a broad range of data on the status and the progression of atherosclerosis, which become incomplete and less precise when restricted ultrasound protocols are used. Although the METEOR results were robust for limitations in the ultrasound protocol, restrictions in the ultrasound procedure may become critical and important in settings in which intervention effects or sample sizes are smaller and a treatment effect could not be observed at all.

\section{Completeness Rates in Previous Studies}

Data to support the difficulties with measurement of the carotid bifurcation and internal carotid artery are very limited, and this view is based largely on results from studies conducted a decade ago. ${ }^{15,21-23}$ For example, measurements of the carotid bifurcation and internal carotid artery were available in only $83 \%$ and $56 \%$ of the individuals in the Rotterdam Study, respectively, whereas measurements of the common carotid artery could be obtained in 97\% of the individuals. ${ }^{23}$ Completeness rates from more recent studies support the use of more extensive protocols, including measurements from the carotid bifurcation and internal carotid artery, because these studies showed a marked improvement in data availability with completeness rates of $\geq 84 \%$ for all carotid sites. ${ }^{9,16,18}$

The completeness rates presented here are similar to completeness rates in the Rating Atherosclerotic Disease Change by Imaging With a New CETP Inhibitor (RADIANCE) trials. ${ }^{18}$ The RADIANCE trials were performed in participants with mixed dyslipidemia and familial hypercholesterolemia, whereas the METEOR results presented here come from a low-risk population. Therefore, the presence and extent of carotid atherosclerosis may seem to be different across these populations and may influence completeness rates. However, showing that completeness can be obtained in a variety of populations with a different level of local atherosclerosis adds to the generalizability of the feasibility of obtaining high completeness rates with extensive ultrasound protocols.

\section{Determinants of Incomplete Data}

Increased BMI was associated with a higher probability for missing CIMT measurements. Such an observation is generally recalled by sonographers and is consistent with the findings in RADIANCE 1 and $2 .^{18}$ The mixed dyslipidemia population in RADIANCE 2 was more overweight than the METEOR and familial hypercholesterolemia population in RADIANCE $1 .{ }^{18}$ This difference in BMI may have caused the lower completeness rate of near wall measurements of the internal carotid artery in patients with mixed dyslipidemia (86\%). Image acquisition may be more difficult in overweight subjects because of greater difficulties in distinguishing near and far wall boundaries on an ultrasound image or because of physical limitations associated with a shorter or thicker neck. The mixed dyslipidemia population may also have more atherosclerotic plaque burden, which could make the arterial walls more difficult to visualize because of increased reflectance from surrounding tissues. Although we found an association between BMI and data availability in the present study, no association between baseline thickness of the CIMT and data completeness was found. This implies that although increased BMI is related to missingness, the missingness could not be explained by the actual thickness of the CIMT. Hence, it is unlikely that a thicker CIMT, reflecting atherosclerotic disease, has been missed in participants with high BMIs. 


\section{Extensive or Restricted Ultrasound Protocols}

A recent consensus statement from the American Society of Echocardiography recommended restricting CIMT ultrasound protocols to measurement of the far wall common carotid artery alone, supplemented by carotid plaque assessment in the common carotid artery, carotid bifurcation, and internal carotid artery. ${ }^{24}$ Although our findings could be used to support assessment of the near wall and the carotid bifurcation and internal carotid artery, these measurements are currently not recommended in the American Society of Echocardiography statement. In addition, the usefulness of CIMT measurements from circumferential angles of insonation and the number of ultrasound examinations over time are additional issues that are relevant for the choice of extensive or restricted ultrasound protocols.

The value of performing near wall CIMT measurements has been debated for many years, because near wall CIMT measurements are an approximation of the true wall thickness. ${ }^{25-27}$ Recent studies have demonstrated that ultrasound protocols assessing both the near and far walls provide the best balance between high reproducibility, large progression rates, and large and precise intervention effects. ${ }^{18,28,29}$ Our findings are in agreement with these studies and suggest that combining near and far wall measurements improve data quality and may add valuable information.

Measurements of the carotid bifurcation and internal carotid artery are also not recommended by the American Society of Echocardiography consensus statement, given the expected difficulties with visualization of these arterial segments. ${ }^{25}$ We showed that CIMT measurements from the internal carotid artery and carotid bifurcation are nearly always complete and that exclusion of these segments may not fully describe the burden of atherosclerosis and the effect of treatment on changes in this burden. This finding is supported by studies showing that progression rates and that the effects of treatment differ across the carotid segments. ${ }^{10,11,30,31}$

The usefulness of CIMT measurements from different circumferential carotid angles has not been extensively studied. A recent study showed that atherosclerosis is asymmetrically distributed across carotid angles within an arterial segment. ${ }^{32}$ Hence, the angle of insonation is an important determinant of the absolute value of maximum CIMT and supports the future use of CIMT measurements from multiple carotid angles. However, we found minor differences in observed treatment effect and its precision with the inclusion of five, three, or one circumferential carotid angle. This may indicate that the rates of change in CIMT are similar across angles of interrogation, even though the absolute values differ per angle.

Besides restrictions in the number of CIMT measurements, the number of ultrasound examinations over time could also be a mean to restrict an ultrasound protocol in longitudinal studies. We recently showed that study designs with a reduced number of ultrasound examinations over time did minimally affect the direction and magnitude of treatment effects on the rate of change in CIMT. ${ }^{33}$ However, a reduced number of exams substantially increased standard errors of rates of change, suggesting that larger sample sizes would be required to have the same level of statistical power.

\section{Implications}

Our study supports the use of extensive ultrasound protocols. However, costs and time investments are clearly higher once choosing an extensive CIMT protocol with assessment of the near and far walls of multiple carotid segments at several angles of insonation. For example, a full METEOR scan took approximately $45 \mathrm{~min}$, whereas a restricted ultrasound protocol with evaluation of the far wall of the common carotid artery at one angle may be performed within $20 \mathrm{~min}$. Nevertheless, high reproducibility, complete data, and assessment of all aspects of carotid atherosclerosis are key reasons to measure CIMT repeatedly across multiple segments and carotid angles. However, the choice of ultrasound protocol and ultrasound equipment should depend on a well-considered balance between time and costs on one hand and data quality and the value of additional information obtained using extensive protocols on the other. ${ }^{34}$ Foremost, as studies of the rate of change in CIMTover time have different issues to address than studies of determinants of CIMT or CIMT as a predictor of risk, the main driving force for ultrasound protocols is of course in relation to the research question at hand.

\section{Future Directions}

The present study showed that high levels of complete data could be obtained using extensive ultrasound protocols. A recent study demonstrated the feasibility of the use of an extensive ultrasound protocol in a screening program for subclinical atherosclerosis. ${ }^{35}$ However, data that directly compare the risk stratification abilities of a far wall common CIMT measurement versus one that uses information from multiple carotid segments are not yet available. These data are important given the growing interest in the use of measures of subclinical atherosclerosis to improve risk stratification for cardiovascular disease events. $^{36-38}$

\section{Limitations}

The availability of CIMT measurements in the present study was examined without further inspection of the correctness of these values. Inclusion of incorrect values could affect the associations found between incompleteness and risk factors. However, the analyses herein were performed on the same data set as was used for the original METEOR publication. ${ }^{10}$ The high quality of the ultrasound equipment in the imaging centers and the core laboratories and the training and continuous quality control used, which resulted in high interreader and intrareader reproducibility, allow us to assume that a substantial effect of incorrect measurements on these results is not likely. Also, the present study was based on analyses of existing data collected during the METEOR trial, which was not primarily designed to address the questions of the current study. Although a prospective study design may have been preferred, we consider it unlikely that the retrospective design used here would have affected the completeness rates. Finally, $1.5 \%$ of the screened individuals were not included in METEOR, because of the inability to determine whether at least one CIMT measurement was $\geq 1.2$ and $<3.5 \mathrm{~mm}$ at two screening examinations. The reasons for ineligibility to determine this criterion were anatomic deviations, including nonvisualized tip of the flow divider, deep vessels, or tortuous vessels. Hence, our results apply only to low-risk individuals in whom visualization of the carotid artery is possible.

\section{CONCLUSIONS}

Extensive ultrasound protocols that include examination of the near walls of the carotid bifurcation and internal carotid artery from multiple angles of interrogation are feasible and can provide high levels of complete CIMT data. Although completeness is high, relatively more CIMT measurements are missing with increasing BMI. Extensive ultrasound protocols are required to obtain the highest precision to observe a treatment effect and to describe the asymmetric nature of atherosclerotic burden in its full range. 


\section{ACKNOWLEDGMENTS}

We would like to thank all the METEOR investigators and the individuals involved in this study: Belgium: Marcelo Goldstein, Anderlecht; Jan Staessen, Leuven; and Guy Marchal, Leuven. Czech Republic: Ales Linhart, Prague. Finland: Jukka T. Salonen, Kuopio. France: Alain Simon, Paris Cedex; Patrick Audouy, Paris; Charles Baranes, Paris; Jean-Philippe Brugnaux, Paris; Eric Chabaud, Ivry sur Seine; Daniel Delbecq, Paris; Jean Claude Mouchet, Meudon; Andre Sebbah, Paris; Gilles Bertrand, Metz; Marcel Frohn, Moutiers; Jean-Luc Jacques, Mars la Tour; Dominique Richter, Jarney; Jean-Louis Doubet, Thionville; Philippe Martin, Yutz; Alain Prochasson, Metz; Eric De Ste Lorette, Paris; JeanClaude Ingrand, Les Lilas; Denis Smila, Yerres; Genevieve Arcizet, Bangolet; Pascal Didi, Paris; Albert Cohen, Les Lilas; Mohamed Oulmekki, Drancy; Karima Allouache, Bangolet; and Serge Kownator, Thionville. Germany: Raimund Erbel, Essen; and Clemens Von Shacky, Munich. Norway: Leiv Ose, Oslo; Knut Risberg, Skedsmokorset; Andreas Tandberg, Bekkestua; Olyvind Aabo, Oslo; Jon Christensen, Oslo; Anne C. Poole, Oslo; and David Russell, Oslo. The Netherlands: Annette Bak, Utrecht; and Jan Jonker, Rotterdam. United States: Thomas W. Littlejohn, Winston-Salem, NC; David M. Capuzzi, Philadelphia, PA; John Morgan, Philadelphia, PA; Robert Davidson, Los Angeles, CA; Michael Davidson, Chicago, IL; Alan Hirsch, Minneapolis, MN; Stephen Glasser, Minneapolis, MN; Leslie Miller, Minneapolis, MN; Daniel Duprez, Minneapolis, MN; Donald Hunninghake, Minneapolis, MN; Robert Knopp, Seattle, WA; Christie Ballantyne, Houston, TX; William Insull, Houston, TX; Daniel Edmundowicz, Pittsburgh, PA; Alan Gradman, Pittsburgh, PA; Neville Bittar, Madison, WI; Laurence Yellen, San Diego, CA; David Cameron, Kirkland, WA; Evan Stein, Cincinnati, $\mathrm{OH}$; Eli Roth, Cincinnati, OH; Tasneem Z. Naqvi, Los Angeles, CA; Cecil Farrington, Salisbury, NC; Bernard Mizok, Chicago, IL; Zane P. Osborne, Chicago, IL; Monica Perlman, La Jolla, CA; Margaret Drehobl, San Diego, CA; Dean Kereiakes, Cincinnati, OH; Norman M. Lunde, Brooklyn Center, MN; Stephen Pomeranz, Cincinnati, OH; Steven Feinstein, Chicago, IL; Charles McCollum, Houston, TX; James Stein, Madison, WI; Laurence Needleman, Philadelphia, PA; Kim Sutton-Tyrell, Pittsburgh, PA; Shirley Otis, La Jolla, CA; and Kirk Beach, Seattle, WA.

From the US CIMT core laboratory at the Wake Forest University School of Medicine, Winston-Salem, NC: Gregory Evans, MA, and Ward Riley, PhD (co-chairs). From the European CIMT core laboratory at the Vascular Imaging Center, Julius Center for Health Sciences and Primary Care, University Medical Center Utrecht, Utrecht, The Netherlands: Anne-Marie Bos, Michiel Bots, MD, PhD (co-chair), Corine van Everdingen, Marrii Geurtsen, Frank Leus, Corry Loffveld, Rudy Meijer, MSc, Dicky Mooiweer-Bogaerdt, Karin Nijssen (co-chair), BSc, Hannie Noordzij, Anneke Rutgers, Els Stooker, Brigitte van der Vlist, Lucienne van der Vlist, Arthur Waterschoot, and Elise Wineke.

\section{REFERENCES}

1. Salonen R, Salonen JT. Determinants of carotid intima-media thickness: a population-based ultrasonography study in eastern Finnish men. J Intern Med 1991;229:225-31

2. Polak JF, O'Leary DH, Kronmal RA, Wolfson SK, Bond MG, Tracy RP, et al. Sonographic evaluation of carotid artery atherosclerosis in the elderly: relationship of disease severity to stroke and transient ischemic attack. Radiology 1993;188:363-70.

3. Howard G, Burke GL, Evans GW, Crouse JR III, Riley W, Arnett D, et al., ARIC Investigators. Relations of intimal-medial thickness among sites within the carotid artery as evaluated by B-mode ultrasound. Stroke 1994;25:1581-7.
4. Bots ML, Hoes AW, Koudstaal PI, Hofman A, Grobbee DE. Common carotid intima-media thickness and risk of stroke and myocardial infarction: the Rotterdam study. Circulation 1997;96:1432-7.

5. Lorenz MW, Markus HS, Bots ML, Rosvall M, Sitzer M. Prediction of clinical cardiovascular events with carotid intima-media thickness: a systematic review and meta-analysis. Circulation 2007; 115:459-67.

6. Espeland MA, O'Leary DH, Terry JG, Morgan T, Evans G, Mudra H. Carotid intimal-media thickness as a surrogate for cardiovascular disease events in trials of HMG-CoA reductase inhibitors. Curr Control Trials Cardiovasc Med 2005;6:3

7. Bots ML, Evans GW, Riley WA, Grobbee DE. Carotid intima-media thickness measurements in intervention studies: design options, progression rates, and sample size considerations: a point of view. Stroke 2003;34: 2985-94.

8. Kastelein JJ, van Leuven SI, Evans GW, Riley WA, Revkin JH, Shear CL, et al. Designs of RADIANCE 1 and 2: carotid ultrasound studies comparing the effects of torcetrapib/atorvastatin with atorvastatin alone on atherosclerosis. Curr Med Res Opin 2007;23:885-94.

9. Bots ML, Evans GW, Riley W, Meijer R, McBride KH, Paskett ED, et al. The Osteoporosis Prevention and Arterial Effects of Tibolone (OPAL) study: design and baseline characteristics. Control Clin Trials 2003;24: 752-75.

10. Crouse JR III, Raichlen JS, Riley WA, Evans GW, Palmer MK, O'Leary DH, et al. Effect of rosuvastatin on progression of carotid intima-media thickness in low-risk individuals with subclinical atherosclerosis: the METEOR Trial. JAMA 2007;297:1344-53.

11. Crouse JR III, Byington RP, Bond MG, Espeland MA, Craven TE, Sprinkle JW, et al. Pravastatin, Lipids, and Atherosclerosis in the Carotid Arteries (PLAC-II). Am J Cardiol 1995; 75:455-9.

12. Blankenhorn DH, Selzer RH, Crawford DW, Barth JD, Liu CR, Liu CH, et al. Beneficial effects of colestipol-niacin therapy on the common carotid artery. Two- and four-year reduction of intima-media thickness measured by ultrasound. Circulation 1993;88:20-8.

13. MacMahon S, Sharpe N, Gamble G, Hart H, Scott I, Simes I, et al., LIPID Trial Research Group. Effects of lowering average of below-average cholesterol levels on the progression of carotid atherosclerosis: results of the LIPID Atherosclerosis Substudy. Circulation 1998;97:1784-90.

14. Salonen IT, Nyyssonen K, Salonen R, Lakka HM, Kaikkonen I, PorkkalaSarataho E, et al. Antioxidant Supplementation in Atherosclerosis Preven tion (ASAP) study: a randomized trial of the effect of vitamins $E$ and $C$ on 3-year progression of carotid atherosclerosis. J Intern Med 2000;248 377-86.

15. Davis PH, Dawson JD, Riley WA, Lauer RM. Carotid intimal-medial thickness is related to cardiovascular risk factors measured from childhood through middle age: the Muscatine Study. Circulation 2001;104:2815-9.

16. Kastelein JJ, Akdim F, Stroes ES, Zwinderman AH, Bots ML, Stalenhoef AF, et al. Simvastatin with or without ezetimibe in familial hypercholesterolemia. N Engl J Med 2008;358:1431-43.

17. Furberg CD, Adams HP Jr., Applegate WB, Byington RP, Espeland MA, Hartwell $T$, et al. Asymptomatic Carotid Artery Progression Study (ACAPS) Research Group. Effect of lovastatin on early carotid atherosclerosis and cardiovascular events. Circulation 1994;90:1679-87.

18. Dogan S, Duivenvoorden R, Grobbee ED, Kastelein JI, Shear CL, Evans GW, et al. Completeness of carotid intima media thickness mea surements depends on body composition: the RADIANCE 1 and 2 trials. J Atheroscler Thromb 2010;17:526-35.

19. Crouse JR III, Grobbee DE, O'Leary DH, Bots ML, Evans GW, Palmer MK, et al. Measuring Effects on Intima Media Thickness: An Evaluation of Rosuvastatin in subclinical atherosclerosis-the rationale and methodology of the METEOR study. Cardiovasc Drugs Ther 2004;18: 231-8.

20. Bates D, Maechler M. lme4: linear mixed-effects models using S4 classes 2010 [Computer software].

21. Chambless LE, Heiss G, Folsom AR, Rosamond W, Szklo M, Sharrett AR, et al. Association of coronary heart disease incidence with carotid arterial wall thickness and major risk factors: the Atherosclerosis Risk in Communities (ARIC) study 1987-1993. Am J Epidemiol 1997;146:483-94. 
22. Espeland MA, Craven TE, Miller ME, D'Agostino R Jr. 1996 Remington lecture: modeling multivariate longitudinal data that are incomplete. Ann Epidemiol 1999;9:196-205.

23. Iglesias del Sol A, Bots ML, Grobbee DE, Hofman A, Witteman JC. Carotid intima-media thickness at different sites: relation to incident myocardial infarction; the Rotterdam Study. Eur Heart J 2002;23:934-40.

24. Stein JH, Korcarz CE, Hurst RT, Lonn E, Kendall CB, Mohler ER, et al. Use of carotid ultrasound to identify subclinical vascular disease and evaluate cardiovascular disease risk: a consensus statement from the American Society of Echocardiography Carotid Intima-Media Thickness Task Force. Endorsed by the Society for Vascular Medicine. J Am Soc Echocardiogr 2008;21: 93-111.

25. Wikstrand J. Methodological considerations of ultrasound measurement of carotid artery intima-media thickness and lumen diameter. Clin Physiol Funct Imaging 2007;27:341-5.

26. Montauban van Swijndregt AD, De Lange EE, de GE, Ackerstaff RG. An in vivo evaluation of the reproducibility of intima-media thickness measurements of the carotid artery segments using B-mode ultrasound. Ultrasound Med Biol 1999;25:323-30.

27. Pignoli P, Tremoli E, Poli A, Oreste P, Paoletti R. Intimal plus medial thickness of the arterial wall: a direct measurement with ultrasound imaging. Circulation 1986;74:1399-406.

28. Dogan S, Plantinga Y, Evans GW, Meijer R, Grobbee DE, Bots ML. Ultrasound protocols to measure carotid intima-media thickness: a post-hoc analysis of the OPAL study. Curr Med Res Opin 2009;25:109-22.

29. Dogan S, Duivenvoorden R, Grobbee DE, Kastelein JJ, Shear CL, Evans GW, et al. Ultrasound protocols to measure carotid intima-media thickness in trials; comparison of reproducibility, rate of progression, and effect of intervention in subjects with familial hypercholesterolemia and subjects with mixed dyslipidemia. Ann Med 2010;42:447-64.

30. Espeland MA, Evans GW, Wagenknecht LE, O'Leary DH, Zaccaro DJ Crouse JR, et al. Site-specific progression of carotid artery intimal-medial thickness. Atherosclerosis 2003;171:137-43.
31. Mackinnon AD, Jerrard-Dunne $\mathrm{P}$, Sitzer $\mathrm{M}$, Buehler $\mathrm{A}$, von $\mathrm{KS}$, Markus HS. Rates and determinants of site-specific progression of carotid artery intima-media thickness: the carotid atherosclerosis progression study. Stroke 2004;35:2150-4.

32. Tajik P, Meijer R, Duivenvoorden R, Peters SA, Kastelein J, Visseren FJ, et al. Asymmetrical distribution of atherosclerosis in the carotid artery: identical patterns across age, race, and gender. Eur J Cardiovasc Prev Rehabil. In press.

33. Peters SA, Palmer MK, Grobbee DE, Crouse JR III, Evans GW, Raichlen JS, et al. Effect of number of ultrasound examinations on the assessment of carotid intima-media thickness changes over time: the example of the METEOR study. J Hypertens 2011;29:1145-54.

34. Baldassarre D, Tremoli E, Amato M, Veglia F, Bondioli A, Sirtori CR. Reproducibility validation study comparing analog and digital imaging technologies for the measurement of intima-media thickness. Stroke 2000; $31: 1104-10$

35. Naqvi TZ, Mendoza F, Rafii F, Gransar H, Guerra M, Lepor N, et al High prevalence of ultrasound detected carotid atherosclerosis in subjects with low Framingham risk score: potential implications for screening for subclinical atherosclerosis. J Am Soc Echocardiogr 2010;23: 809-15.

36. Lorenz MW, Schaefer C, Steinmetz H, Sitzer M. Is carotid intima media thickness useful for individual prediction of cardiovascular risk? Ten-year results from the Carotid Atherosclerosis Progression Study (CAPS). Eur Heart J 2010;31:2041-8

37. Nambi V, Chambless L, Folsom AR, He M, Hu Y, Mosley T, et al. Carotid intima-media thickness and presence or absence of plaque improves prediction of coronary heart disease risk: the ARIC (Atherosclerosis Risk in Communities) study. J Am Coll Cardiol 2010; 55:1600-7.

38. Xie W, Wu Y, Wang W, Zhao D, Liang L, Wang M, et al. A longitudinal study of carotid plaque and risk of ischemic cardiovascular disease in the Chinese population. J Am Soc Echocardiogr 2011;24:729-37.

\section{Did you know?}

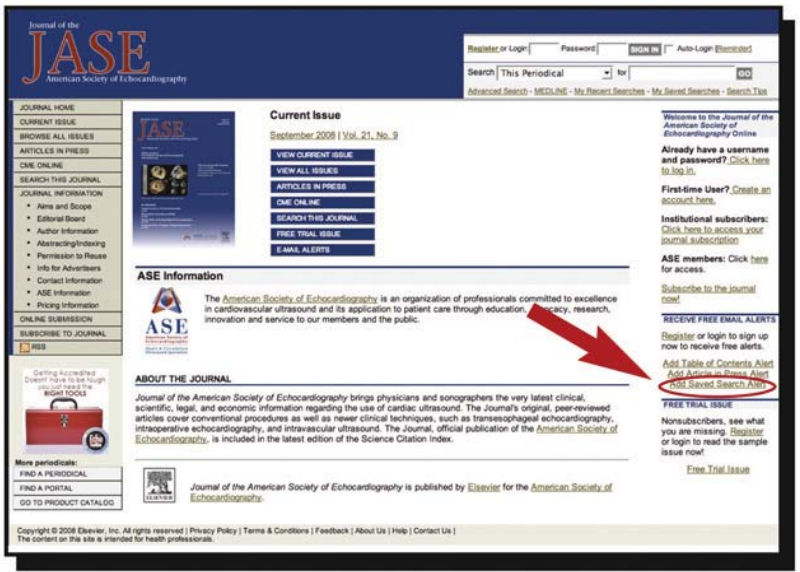

You can save your online searches and get the results by email.

\section{Visit www.onlinejase.com today!}

